\title{
Terapia nutricional em pacientes com COVID-19: algumas considerações e evidências científicas
}

\author{
Nutritional therapy in patients with COVID-19: some considerations and scientific evidence \\ Terapia nutricional en pacientes con COVID-19: algunas consideraciones y evidencia científica
}

Recebido: 28/02/2021 | Revisado: 08/03/2021 | Aceito: 14/03/2021 | Publicado: 20/03/2021

Beatriz Souza Santos

ORCID: https://orcid.org/0000-0002-2464-4674 Universidade Federal do Piauí, Brasil

E-mail: santosbsnutri@gmail.com

Camila Santos Marreiros

ORCID: https://orcid.org/0000-0001-6225-1698

Universidade Federal do Piauí, Brasil

E-mail: camila.marreiros@ hotmail.com

Fernanda Kassiely de Sousa Veloso

ORCID: https://orcid.org/0000-0002-5289-2624 Universidade Federal do Piauí, Brasil

E-mail: f.kassiely@gmail.com

Jardel Alves da Costa

ORCID: https://orcid.org/0000-0002-9844-0770 Universidade Federal do Piauí, Brasil

E-mail: jardelalves@ufpi.edu.br

Karine Rodrigues Ferreira

ORCID: https://orcid.org/0000-0002-4140-3119

Universidade Federal do Piauí, Brasil

E-mail: karinerodriguesf9@gmail.com

Lídia Ribeiro de Carvalho

ORCID: https://orcid.org/0000-0003-3267-4002

Universidade Federal do Piauí, Brasil

E-mail: lidiahupd@gmail.com

Renata Rodrigues Costa Fontinele

ORCID: https://orcid.org/0000-0002-6121-0322

Universidade Federal do Piauí, Brasil

E-mail: renatafontinele25@hotmail.com

Stefany Dourado da Silva

ORCID: https://orcid.org/0000-0002-1860-2486

Universidade Federal do Piauí, Brasil

E-mail: stefanyparamore@hotmail.com

Gilmara Péres Rodrigues

ORCID: https://orcid.org/0000-0003-2770-8191

Universidade Federal do Piauí, Brasil

E-mail: gilmaraperes@ufpi.edu.br

\begin{abstract}
Resumo
O presente estudo objetivou revisar a literatura sobre a terapia nutricional em pacientes com COVID-19, destacando alguns nutrientes com potencial funcional que podem beneficiar os pacientes na resposta à infecção e recuperação da doença. Trata-se de uma revisão narrativa simples, desenvolvida a partir de levantamento bibliográfico, leitura de artigos originais e de outras revisões sobre a temática. Os artigos foram recuperados a partir das bases de dados PubMed, Scielo e Web of Science. Os principais achados mostraram que a desnutrição é característica frequente em pacientes infectados por SARS-CoV-2, estando associada à morbidade e mortalidade aumentadas. As evidências científicas confirmam a terapia nutricional como determinante no prognóstico do paciente com COVID-19, com recomendações para oferta hiperproteica e teor adequado de nutrientes com potencial funcional, como os considerados imunomoduladores e anti-inflamatórios. Conclui-se que a terapia nutricional com oferta aumentada de proteínas e rica em imunonutrientes, pode melhorar o prognóstico clínico de pacientes com COVID-19.
\end{abstract}

Palavras-chave: COVID-19; Nutrição; Imunonutrição; Biomarcadores nutricionais. 


\begin{abstract}
The present study aimed to review the literature on nutritional therapy in patients with COVID-19, highlighting some nutrients with functional potential that can benefit patients in responding to infection and recovery from the disease. It is a simple narrative review, developed from a bibliographic survey, reading of original articles and other reviews on the theme. The articles were retrieved from the PubMed, Scielo and Web of Science databases. The main elevated findings are that malnutrition is a frequent feature in patients infected with SARS-CoV-2, associated with increased morbidity and mortality. Scientific evidence confirms nutritional therapy as a determinant in the prognosis of patients with COVID-19, with recommendations for hyperprotein supply and adequate content of nutrients with functional potential, such as those considered immunomodulatory and anti-inflammatory. It is concluded that a nutritional therapy with an increased supply of protein and rich in immunonutrients, can improve the clinical prognosis of patients with COVID-19.
\end{abstract}

Keywords: COVID-19; Nutrition; Immunonutrition; Nutritional biomarkers.

\title{
Resumen
}

El presente estudio tuvo como objetivo revisar la literatura sobre terapia nutricional en pacientes con COVID-19, destacando algunos nutrientes con potencial funcional que pueden beneficiar a los pacientes en la respuesta a la infección y la recuperación de la enfermedad. Se trata de una simple revisión narrativa, desarrollada a partir de un relevamiento bibliográfico, lectura de artículos originales y otras revisiones sobre el tema. Los artículos fueron recuperados de las bases de datos PubMed, Scielo y Web of Science. Los principales hallazgos elevados son que la desnutrición es una característica frecuente en pacientes infectados con SARS-CoV-2, asociada con una mayor morbilidad y mortalidad. La evidencia científica confirma la terapia nutricional como determinante en el pronóstico de los pacientes con COVID-19, con recomendaciones de aporte de hiperproteínas y contenido adecuado de nutrientes con potencial funcional, como los considerados inmunomoduladores y antiinflamatorios. Se concluye que una terapia nutricional con mayor aporte proteico y rica en inmunonutrientes, puede mejorar el pronóstico clínico de los pacientes con COVID-19.

Palabras clave: COVID-19; Nutrición; Inmunonutrición; Biomarcadores nutricionales.

\section{Introdução}

Em dezembro de 2019, a China relatou a ocorrência de vários casos de pneumonia de etiologia desconhecida, com exposição comum. Em 7 de janeiro de 2020, as autoridades chinesas identificaram um novo tipo de vírus na família Coronaviridae como agente causador do surto da Síndrome Respiratória Aguda Grave - Coronavírus - 2 (SARS-CoV-2). A partir de então, o surgimento repentino, a rápida taxa de contágio e evolução, bem como os impactos sociais, econômicos e no sistema de saúde, têm assustado o Brasil e todo o mundo (Wang et al., 2020a).

O SARS-COV-2 é um vírus respiratório que provoca síndrome respiratória aguda, com potencial inflamatório. A sintomatologia clínica é diversa e pode incluir febre, tosse, dispneia, fadiga, cefaleia, odinofagia, ageusia, anosmia, náuseas, coriza, diarreia, além de outros sintomas respiratórios e multissistêmicos. Na Síndrome Respiratória Aguda Grave (SARS) por COVID-19, o aumento sérico de citocinas pró-inflamatórias foi associado à extensa infiltração pulmonar de neutrófilos e necessidade de ventilação mecânica (Wang et. al., 2020b).

Evidências científicas têm mostrado que indivíduos idosos, maiores de 60 anos, ou mesmo indivíduos de qualquer idade, portadores de doenças crônicas, como síndrome metabólica, doenças cardiovasculares, obesidade, hipertensão, diabetes, hipovitaminose D, asma, doenças pulmonares crônicas e insuficiência renal, são os mais propensos ao surgimento de complicações por COVID-19. Nessas pessoas, observa-se alto índice de hospitalização devido à ocorrência frequente de complicações respiratórias e da necessidade de suporte de oxigênio, aumentando a taxa de mortalidade pela doença (Szklo, 2020; Costa et al., 2020; De Lucena, 2020).

A obesidade tem se destacado como potencializadora do risco de complicações por COVID-19, por ser base fisiopatológica para a inflamação crônica de baixo grau, condição favorável ao desenvolvimento das outras comorbidades. O papel metabólico do tecido adiposo envolve a síntese de citocinas inflamatórias, entre as quais os fatores de necrose tumoral (TNF- $\alpha$ e TNF- $\beta$ ), interleucinas (IL-4, IL-6, IL-10, IL-1 $\beta$ ), interferons, quimiocinas e fator de transformação do crescimento beta (TGF- $\beta$ ) (Kassir, 2020; Ryan \& Caplice, 2020). 
A superexpressão dessas adipocitocinas inflamatórias nos depósitos de gordura visceral afetam a resposta imunológica, prejudicam a quimiotaxia e modificam a diferenciação de macrófagos. Diretamente relacionado à infecção por COVID-19, o tecido adiposo funciona como reservatório do vírus, provavelmente devido à capacidade dos adipócitos de sintetizar IL-6 e seu receptor (IL-6R), sinalizando a cascata inflamatória (Kassir, 2020).

No extremo oposto, pacientes com desnutrição também estão mais vulneráveis ao desenvolvimento das formas graves da doença. Nesses pacientes, há prejuízo da resposta imunológica, maior suscetibilidade à infecção e alto risco de mortalidade (Farhadi \& Ovchinnikov, 2018; Alwarawhah et al., 2018; Handu et al., 2020). Em ambas as condições nutricionais, de excesso ou de déficit de peso corporal, pode existir desnutrição e deficiência de micronutrientes importantes para a resposta imunológica e capacidade orgânica de recuperar-se do estresse metabólico provocado pela infecção.

Nesse contexto, o presente estudo objetivou revisar a literatura sobre a terapia nutricional em pacientes com COVID-19, destacando alguns nutrientes com potencial imunomodulador, que podem beneficiar os pacientes na resposta à infecção e recuperação da doença.

\section{Metodologia}

Trata-se de uma revisão bibliográfica do tipo narrativa simples, realizada por meio de pesquisa nas bases de dados PubMed, Scielo e Web of Science. Foram selecionadas publicações em português, inglês e espanhol, relativas às infecções respiratórias, nutrientes com potencial imunomodulador e anti-inflamatório, e sobre terapia nutricional e pandemia de COVID19. Os descritores utilizados para a busca de artigos foram: COVID-19, SARS-CoV-2, coronavírus, intervenção nutricional, biomarcadores nutricionais, imunonutrição e micronutrientes.

\section{Resultados e Discussão}

\subsection{Principais biomarcadores de gravidade na COVID-19}

Inúmeras citocinas pró-inflamatórias (IL-6, IL-1 $\beta$, IL-8, TNF $\alpha$ ) têm sido descritas como importantes no processo fisiopatológico da síndrome respiratória aguda grave (SARS) (Matute-Bello et al., 2008; Lindauer et al., 2009). Na COVID-19, a resposta inflamatória sistêmica provocada pelo SARS-CoV-2 desencadeia elevações nas concentrações de citocinas relacionadas à injúria multissistêmica. $\mathrm{O}$ aumento de troponina é acompanhado por elevação de outros marcadores inflamatórios, como D-dímero, ferritina, interleucina-6 (IL-6), desidrogenase láctica (DHL), proteína C reativa, pro-calcitonina e contagem de leucócitos, identificando a fase inflamatória da doença caracteriza pela tempestade de citocinas (Clerkin et al., 2020; Shi et al., 2020; Zheng et al., 2020).

Pacientes com alterações significativas dos marcadores supramencionados têm pior prognóstico da doença e alta taxa de mortalidade. Shi et al. (2020) avaliaram 416 pacientes e observaram que o aumento da mortalidade e a SRAS está associada à injúria miocárdica. Zhou et al. (2020) mostraram que pacientes que evoluíram para o óbito apresentaram níveis mais altos de IL-6, ferritina e DHL, bem como linfopenia. Os resultados deste estudo sugerem que valores de D-dímero acima de $1 \mu \mathrm{g} / \mathrm{mL}$ na admissão hospitalar é preditor independente de mortalidade.

Além disso, marcadores de disfunção miocárdica também têm sido identificados como indicadores de mau prognóstico em pacientes com COVID-19, devido ao comprometimento da função cardíaca e maior necessidade de ventilação mecânica. Guan et al. (2020) mostraram que 27,8\% de 187 pacientes com COVID-19 apresentaram elevação de troponina, com aumento na taxa de óbito de 7,6\% para 37,5\% em pacientes sem doenças cardiovasculares (DC) e de 13,3\% para 69,4\% naqueles já portadores de DC.

Níveis elevados de troponina também foram correlacionados ao aumento nas concentrações de proteína $C$ reativa e de NT-proBNP (fragmento N-terminal do peptídeo natriurético tipo B), indicadores de insuficiência cardíaca (Lippi et al., 2020). 
Esses achados foram corroborados por metanálise composta por 342 pacientes com COVID-19 nas formas moderada e grave da doença (Wang et al., 2020).

\subsection{Estado nutricional e nutrientes com potencial imunomodulador}

A desnutrição é característica frequente em pacientes com SARS, estando associada à morbidade e mortalidade aumentadas. Em pacientes com insuficiência respiratória, o gasto energético aumentado é acompanhado pela inapetência, dispneia e pelas alterações metabólicas que influenciam o metabolismo dos nutrientes favorecendo a perda excessiva de peso e massa corporal (SBNPE, 2011; Girão et al., 2020).

Apesar de não ser capaz por si só, de impedir o ciclo vicioso de degradação e fadiga muscular, a terapia nutricional, associada à reabilitação pulmonar, correção da hipóxia, terapia medicamentosa, controle inflamatório e mudanças nos hábitos, é fundamental para o sucesso do tratamento e restabelecimento do paciente (SBNPE, 2011; Dellalibera-Joviliano, Dellalibera \& Fujita, 2020). O impacto da desnutrição sobre a capacidade do sistema imunológico e da resposta orgânica à infecção é estabelecido na literatura (Beck et al., 2004; Dias et al., 2020; Feiber, 2015; Francisco et al., 2006; Schaible \& Kaufmann, 2007).

Assim, para além da desnutrição, as deficiências nutricionais têm sido associadas a maior propensão para infecções virais, bem como para a ocorrência/manutenção de estados metabólicos de inflamação e estresse oxidativo. Nutrientes imunomoduladores são aqueles capazes de ativar linfócitos e macrófagos, produzir moléculas vasodilatadoras, inibir a função neutrofílica e estimular a produção humoral. Deste grupo, estão destacados nesta revisão as vitaminas A, D e E, o mineral zinco e os probióticos (Elmadfa \& Meyer, 2019).

A vitamina A, usualmente referida como retinoide, atua na renovação de células epiteliais e na produção fisiológica de muco nos tratos respiratórios e intestinais, auxiliando na função imune contra patógenos. A hipovitaminose A tem sido associada ao aumento do risco de infecção por micobactérias, com provável mecanismo de ação que envolve a redução da atividade das células NK (natural killer) e estímulo de mitógenos, com consequente aumento na síntese de interferon pelas células esplênicas e redução da produção de anticorpos (El Beitune et al., 2003; Jason et al., 2002).

Além disso, a hipovitaminose A resulta em alterações nas funções dos linfócitos T e B, e em diminuição na quantidade de células $\mathrm{CD}^{+}$. Essas alterações ocorrem em resposta ao aumento da expressão gênica de interferon gama (IFN- $\gamma$ ) e à diminuição de interleucinas anti-inflamatórias (Biasebetti, Rodrigues \& Mazur, 2018).

A vitamina D está envolvida em muitos processos inflamatórios imunomediados. Entretanto, o impacto dos níveis de vitamina D na resposta imunogênica às infecções ainda não é clara (Gois et al., 2017). Acredita-se que o papel imunomodulador da vitamina $\mathrm{D}$ se deve à alta afinidade entre a forma ativa 25-di-hidroxivitamina $\mathrm{D}_{3}\left(1,25(\mathrm{OH})_{2} \mathrm{D}_{3}\right)$ e o receptor de vitamina D (VDR). Como fator de transcrição, o VDR liga-se a regiões genômicas acessíveis em genes-alvo, regulando o metabolismo, a fim de apoiar o sistema imunológico com energia, e modulando a imunidade inata e adquirida (Mahan et al., 2013; Koivisto et al., 2020).

Um dos prováveis mecanismos de ação envolve a síntese de $1,25(\mathrm{OH})_{2} \mathrm{D}_{3}$ por células do sistema imune em resposta à infecção, permitindo interações diretas e indiretas entre as vias metabólicas e o sistema imunológico do hospedeiro (Khoo et al., 2012). As funções imunorreguladoras da vitamina D incluem a supressão da resposta imune inata, por meio da regulação negativa de citocinas inflamatórias e da polarização do sistema imunológico adaptativo em relação às respostas $\mathrm{T}$ helper-2 (Lang et al., 2013).

A vitamina $\mathrm{E}$, nas formas químicas tocotrienóis e tocoferóis, destaca-se pelo potencial antioxidante, protetor das membranas celulares lipoproteicas, evitando degradação oxidativa e danos gerados pelo excesso de radicais livres (Jordão et al., 2021). Os efeitos imunomoduladores da vitamina E são considerados benéficos na mitigação de várias doenças virais, 
bacterianas e alérgicas, como a asma, possuindo capacidade de modulação da resposta imune inata nas infecções que resultam em pneumopatias (Lima et al.,2020).

Em estudo randomizado, duplo-cego e controlado conduzido em 617 idosos institucionalizados, com idade mínima de 65 anos, realizou-se suplementação diária de 200 UI de vitamina E, durante um ano, contendo metade da dose recomendada diariamente para as demais vitaminas e minerais essenciais. Os resultados mostraram menor ocorrência de infecções respiratórias e resfriados comuns entre os idosos suplementados com vitamina E, embora não tenha sido observada diferença no número de dias com a doença (Calder et al., 2020).

Outro nutriente importante, o zinco $(\mathrm{Zn})$ se destaca como oligoelemento essencial a diversos processos celulares, como síntese de proteínas, reparo de DNA e citoproteção (Skalny, 2020). O potencial do Zn contra processos infecciosos é evidenciado na literatura científica, a exemplo da capacidade do mineral de bloquear a reprodução do vírus da hepatite $\mathrm{E}$, inibindo a polimerase dependente de RNA viral (RdRp). Essa enzima também desempenha importante papel na replicação de alguns coronavírus (Kaushik et al., 2017), o que sugere um potencial promissor do Zn no manejo terapêutico de pacientes infectados pelo SARS-CoV-2.

Sobre o sinergismo entre Zn e fármacos utilizados na COVID-19 (cloroquina e hidroxicloroquina), foi mostrado que a cloroquina pode induzir o aumento das concentrações de Zn no citosol celular, o que aumentaria as chances de atuação do mineral contra a polimerase viral dependente de RNA viral (Shittu, 2020). Esses achados são importantes, considerando as concentrações séricas persistentemente baixas de Zn em pacientes críticos (Hoeger et al., 2017).

No que se refere aos probióticos, o eixo bidirecional intestinal-pulmão, por meio da circulação sanguínea, afeta a saúde pulmonar e a saúde intestinal em trocas que envolvem metabólitos microbianos e endotoxinas, bem como agentes infecciosos instalados nas vias pulmonares (Dumas et al., 2018). Assim, o SARS-CoV-2, promotor de infecção pulmonar por ligação direta aos receptores alveolares ACE2 (enzima conversora de angiotensina 2), também alcança a luz intestinal, tendo sido identificado nas fezes de pacientes com COVID-19. Isso é possível porque, de modo semelhante, as células epiteliais intestinais, particularmente os enterócitos do intestino delgado, também expressam receptores da ACE2 (Zuo et al., 2020).

Em relação ao potencial imunomodulador do probiótico atuando entre intestino-pulmão, um estudo randomizado, duplo-cego, e controlado por placebo avaliou o uso de Lactobacillus GG (LGG) durante 3 meses, os resultados mostraram que as crianças do grupo LGG tiveram um risco significativamente menor de desenvolverem infecções do trato respiratório superior. Quando a infecção ocorria, apresentava menor duração (em dias) e menos sintomas respiratórios, em comparação às infecções desenvolvidas pelas crianças do grupo placebo (Hojsak et al., 2010).

Em adição, Berggren et al. (2011) também confirmaram o potencial imunomodulador dos probióticos na prevenção de infecções respiratórias, por meio de estudo randomizado, paralelo, duplo-cego e controlado por placebo. Neste desenho de estudo, foi investigado se a ingestão de uma mistura probiótica por 12 semanas poderia reduzir o risco de episódios de resfriado comum, gravidade, frequência, número de dias com sintomas, e resposta imune celular em infecções pelo vírus do resfriado comum.

Os resultados mostraram que a incidência de um ou mais episódios de resfriado comum foi reduzida de $67 \%$ no grupo controle para 55\% no grupo probiótico. Além disso, o número de dias com sintomas comuns de resfriado foi reduzido, significativamente, de 8,6 dias no grupo controle para 6,2 dias no grupo probiótico (Berggren et al., 2011).

\subsection{Terapia nutricional em pacientes com COVID-19}

O primeiro passo no tratamento nutricional de pacientes com COVID-19 consiste na avaliação do risco de desnutrição de todos os infectados, ainda na admissão hospitalar, para implantação de suporte nutricional imediato, adequado ao estado de 
nutrição individual. Em pacientes infectados pelo SARS-CoV-2, a ingestão proteica tem sido identificada como fator determinante de evolução nutricional e prognóstico clínico (Zhang \& Liu, 2020).

As recomendações vigentes determinam que, mesmo naqueles pacientes que não correm risco de desnutrição, a oferta deve ser hiperproteica $(1,5 \mathrm{~g} / \mathrm{Kg}$ de peso/ dia), com calorias em torno de 25 a $30 \mathrm{Kcal} / \mathrm{Kg}$ de peso/ dia. Em adição, a dieta deve ser enriquecida em vitaminas e nutrientes com propriedades imunomoduladoras, anti-inflamatórias e antioxidantes (Jin et al., 2020).

Para alcançar essas recomendações, o uso de suplementos nutricionais orais (SNO) tem sido estratégia adotada com boa eficácia em pacientes com COVID-19, sendo necessária devido à inapetência e dificuldade de alimentar-se por via oral provocados pela dispneia e desconforto respiratório. A estratégia nutricional consiste em oferecer os SNO entre as refeições ou imediatamente após as mesmas (Jin et al., 2020).

Para pacientes em risco nutricional, recomendam-se dois a três frascos (125 a $200 \mathrm{~mL} /$ dia) de $\mathrm{SNO}$ proteico-calórico, contendo, respectivamente, 600 a $900 \mathrm{Kcal} /$ dia e 35 a 55 g/ dia. Se o suplemento oral não for tolerado e/ou as condições respiratórias continuarem a se agravar, deve-se prescrever nutrição enteral ou parenteral suplementar/total (NP), planejando contínua monitoração bioquímica (Caccialanza et al., 2020).

A NE intragástrica pode ser fornecida com segurança em pacientes com COVID-19, mesmo com o uso de posicionamento propenso e oxigenação por membrana extracorpórea. Não havendo condições de alimentação por via oral, a NE deve ser iniciada precocemente após a admissão na unidade de terapia intensiva (UTI), recomendando-se fórmula polimérica isosmolar padrão, iniciada em doses tróficas, ajustando-se conforme necessidade do paciente, enquanto se monitora instabilidade hemodinâmica, distúrbios metabólicos e intolerância gastrointestinal. (Martindale et al., 2020).

Em pacientes com intolerância gastrointestinal, alto risco de aspiração ou aumento do suporte vasopressor, é aconselhável a NP. Em pacientes com distensão gástrica e gastrite erosiva, devem ser considerados o uso de NP suplementar ou de fórmulas entéricas altamente digeríveis, específicas e hiperproteicas, enriquecidas com ácidos graxos $\omega-3$ e/ou com outros nutrientes anti-inflamatórios e imunomoduladores, a exemplo do Zn e vitamina D (Calder et al., 2010; Poveda et al., 2018). Ressalta-se que na COVID-19, a decisão por instituir a NP deve ser cautelosa, bem como deve ser prioridade oferecer imunonutrientes por esta via, como tentativa de compensar o seu efeito deletério na morfologia e resposta imune (Gutiérrez et al., 2019; Huang et al., 2020).

$\mathrm{Na}$ terapia nutricional devem ser reforçadas as práticas de controle sanitário, evitando aumento da exposição ou contaminação dos equipamentos, a exemplo do uso de volumes residuais gástricos para monitoramento, calorimetria indireta, endoscopia ou fluoroscopia para obter acesso enteral ou transporte para fora da UTI com fins de obtenção de imagens adicionais. Além disso, as estratégias de terapia nutricional precisam ser avaliadas continuamente, com base no risco/benefício para o paciente e profissionais de saúde (Martindale et al., 2020).

\section{Considerações Finais}

Nesta revisão, buscou-se descrever parte das evidências científicas sobre a terapia nutricional em pacientes com COVID-19, destacando o papel imunomodulador e anti-inflamatório das vitaminas A, D e E, do mineral Zn e de probióticos como complementos dietéticos. Com base no material analisado para esta síntese, conclui-se que a terapia nutricional com oferta aumentada de proteínas e rica em imunonutrientes pode melhorar o prognóstico clínico do paciente com COVID-19.

Nesse contexto, destaca-se que a intervenção precoce com terapia nutricional planejada e adequada ao estado clínico e de nutrição do paciente, pode conter os efeitos prejudiciais do catabolismo, minimizando a degradação muscular e fadiga corporal. As recomendações nutricionais devem considerar a situação clínica do paciente, a presença de complicações metabólicas provocadas pelo SARS-CoV-2, o comprometimento sistêmico e a gravidade dos sintomas. Além disso, é 
importante destacar que o tratamento do paciente com COVID-19 envolve diversos profissionais de saúde, para que melhores resultados sejam alcançados em menor tempo de internação hospitalar e com menos sequelas para o paciente.

Ressalta-se que a presente revisão narrativa da literatura não pretendeu abranger todas as evidências científicas sobre o tema "terapia nutricional em pacientes com COVID-19", devido à complexidade e velocidade com que se investiga e produz conhecimentos nesta abordagem. A pretensão dos autores, portanto, foi apresentar uma síntese confiável que possa direcionar os profissionais de saúde em suas condutas na prática clínica cotidiana.

Assim, recomenda-se que revisões sistemáticas com e sem metanálise sejam realizadas para apresentar, com maior nível de evidência, os efeitos dos nutrientes, isolados ou em combinação, sobre o prognóstico de pacientes infectados pelo novo coronavírus.

\section{Referências}

Alwarawrah, Y., Kiernan, K., \& MacIver, N. J. (2018). Changes in Nutritional Status Impact Immune Cell Metabolism and Function. Frontiers in immunology, 9, 1055. 10.3389/fimmu.2018.01055

Beck, M. A., Handy, J., \& Levander, O. A. (2004). Host nutritional status: the neglected virulence factor. Trends in Microbiology, 12 (9), 417-423. 10.1016/j.tim.2004.07.007

Berggren, A., Lazou Ahrén, I., Larsson, N., \& Önning, G. (2011). Randomised, double-blind and placebo-controlled study using new probiotic lactobacilli for strengthening the body immune defence against viral infections. European Journal of Nutrition, 50 (3), 203-210. org/10.1016/j.tim.2004.07.007

Biasebetti, M. D. B. C., Rodrigues, I. D., \& Mazur, C. E. (2018). Relação do consumo de vitaminas e minerais com o sistema imunitário: uma breve revisão. Visão Acadêmica, 19 (1), 130-136. 10.5380/ACD.V19I1.57737

Caccialanza, R., Laviano, A., Lobascio, F., Montagna, E., Bruno, R., Ludovisi, S., Corsico, A. G., Di Sabatino, A., Belliato, M., Calvi, M., Iacona, I., Grugnetti, G., Bonadeo, E., Muzzi, A., \& Cereda, E. (2020). Early nutritional supplementation in non-critically ill patients hospitalized for the 2019 novel coronavirus disease (COVID-19): Rationale and feasibility of a shared pragmatic protocol. Nutrition, 74, 110835. 10.1016/j.nut.2020.110835

Calder, P. (2010). Rationale and use of n-3 fatty acids in artificial nutrition. Proceedings of the Nutrition Society, 69 (4), 565-573. $10.1017 / \mathrm{S} 0029665110000157$

Calder, P. C., Carr, A. C., Gombart, A. F., \& Eggersdorfer, M. (2020). Optimal nutritional status for a well-functioning immune system is an important factor to protect against viral infections. Nutrients, 12 (4), 1181. 10.3390/nu12041181

Clerkin, K. J., Fried, J. A., Raikhelkar, J., Sayer, G., Griffin, J. M., Masoumi, A., Jain, S. S., Burkhoff, D., Kumaraiah, D., Rabbani, L., Schwartz, A., \& Uriel, N. (2020). COVID-19 and Cardiovascular Disease. Circulation, 141 (20), 1648-1655. 10.1161/CIRCULATIONAHA.120.046941

Costa, F. F et al. (2020). Metabolic syndrome and COVID-19: An update on the associated comorbidities and proposed therapies. Diabetes \& Metabolic Syndrome: Clinical Research \& Reviews, 14 (5), 809-814. 10.1016/j.dsx.2020.06.016

De Lucena, T., Silva Santos, A. F., Lima, B. R., de Albuquerque Borborema, M. E., \& de Azevêdo Silva, J. (2020). Mechanism of inflammatory response in associated comorbidities in COVID-19. Diabetes \& Metabolic Syndrome, 14 (4), 597-600. doi:org/10.1016/j.dsx.2020.05.025

Dellalibera-Joviliano, R., Alves, N. P., Dellalibera, E., \& Fujita, A. T. (2020). Perfil celular imunológico e o papel na resposta inflamatória frente ao SARSCoV-2. Revista Interdisciplinar de Saúde e Educação, 1 (2), 192-207.

Dias, V. M. C. H., Carneiro, M., Vidal, C. F. L., Corradi, M. F. D. B., Brandão, D., Cunha, C. A., Chebabo, A., Oliveira, P. R. D. O., Michelin, L., Rocha, J. L. L., Waib, L. F., Carrilho, C. M., Lobo, S. M. A., Oliveira, M. C. O., Nunes, R. R., Diego, L. A. S., Santos, A. S., Muglia, V., Jr Souza, A. S., Escuissato, D., Neto, C. A., Chatkin, J. M., Martins, R., Maurici, R., Costa, S. F., Alves, J. S., Nascimento, M. M., \& Moura-Neto, J. (2020). Orientações sobre diagnóstico, tratamento e isolamento de pacientes com COVID-19. J. Infect. Control, 9 (2), 56-75. https://jic-abih.com.br/index.php/jic/article/view/295

Dumas, A., Bernard, L., Poquet, Y., Lugo-Villarino, G., \& Neyrolles, O. (2018). The role of the lung microbiota and the gut-lung axis in respiratory infectious diseases. Cellular Microbiology, 20 (12), 12966. doi:org/10.1111/cmi.12966

El Beitune, P., Duarte, G., Nunes de Morais, E., Quintana, S. M., \& Vannucchi, H. (2003). Deficiência da vitamina a e associações clínicas: revisão. Archivos Latinoamericanos de Nutricion, 53 (4), 355-363. http://ve.scielo.org/scielo.php?script=sci_arttext\&pid=S0004 06222003000400004\&lng=es\&tlng=pt

Elmadfa, I., \& Meyer, A. L. (2019). The Role of the Status of Selected Micronutrients in Shaping the Immune Function. Endocrine, Metabolic \& Immune Disorders Drug Targets, 19 (8), 1100-1115. org/10.2174/1871530319666190529101816

Farhadi, S., \& Ovchinnikov, R. S. (2018). The relationship between nutrition and infectious diseases: A review. Biomedical and Biotechnology Research Journal, 2, 168 - 172. org/10.4103/bbrj.bbrj_69_18

Feiber, L. T. (2015). Associação entre parâmetros nutricionais e inflamatórios e o tempo em ventilação mecânica de pacientes pediátricos graves com insuficiência respiratória (Dissertação de mestrado). Universidade Federal de Santa Catarina, Florianópolis, Santa Catarina, Brasil. 
Francisco, P. M. S. B., Donalisio, M. R., Barros, M. B. A., César, C. L. G., Carandina, L., \& Goldbaum, M. (2006). Fatores associados à vacinação contra a influenza em idosos. Rev Panam Salud Publica. 19 (4), 259-64. http://www.scielosp.org/scielo.php?script=sci_arttext\&pid=S1020-49892006000400006

Girão, M. M. F., Paiva Coelho, N., Barroso, B. S., \& Gadelha, M. D. S. V. (2020). Perfil Epidemiológico dos Pacientes de SARS-COV-2 no Brasil. Revista de Psicologia, 14 (51), 646-658.

Guan W-Jie., Ni, Z-yi., Hu Y, Liang W-hua., Ou, J. He, L. Liu, H. Shan, C. Lei, D.S.C. Hui, Guo, T., Fan, Y., Chen, M., Wu, X., Zhang, L., He, T., Wang, H., Wan, J., Wang, X., \& Lu, Z. (2020). Cardiovascular Implications of Fatal Outcomes of Patients With Coronavirus Disease 2019 (COVID-19). JAMA Cardiology, 5 (7), 811-818. doi:org/10.1001/jamacardio.2020.1017

Gutiérrez, S., Svahn, S. L., \& Johansson, M. E. (2019). Effects of Omega-3 Fatty Acids on Immune Cells. International Journal of Molecular Sciences, 20 (20), 5028. doi:org/10.3390/ijms20205028

Handu, D., Moloney, L., Rozga, M., \& Cheng, F. (2020). Malnutrition Care during the COVID-19 Pandemic: Considerations for Registered Dietitian Nutritionists Evidence Analysis Center. Journal of the Academy of Nutrition and Dietetics. doi:10.1016/j.jand.2020.05.012

Hoeger, J., Simon, T. P., Beeker, T., Marx, G., Haase, H., \& Schuerholz, T. (2017). Persistent low serum zinc is associated with recurrent sepsis in critically ill patients - A pilot study. PloS one. 12 (5), e0176069. doi:org/10.1371/journal.pone.0176069

Hojsak, I et al. (2010). Lactobacillus GG in the prevention of gastrointestinal and respiratory tract infections in children who attend day care centers: a randomized, double-blind, placebo-controlled trial. Clinical Nutrition, 29 (3), 312-316. doi:org/10.1016/j.clnu.2009.09.008

Huang, C., Wang, Y., Li, X., Ren, L., Zhao, J., Hu, Y., Zhang, L., Fan, G., Xu, J., Gu, X., Cheng, Z., Yu, T., Xia, J., Wei, Y., Wu, W., Xie, X., Yin, W., Li, H., Liu, M., Xiao, Y., \& Cao, B. (2020). Clinical features of patients infected with 2019 novel coronavirus in Wuhan, China. Lancet, 395 (10223), 497-506. org/10.1016/S0140-6736(20)30183-5

Jason, J., Archibald, L. K., Nwanyanwu, O. C., Sowell, A. L., Buchanan, I., Larned, J., Bell, M., Kazembe, P. N., Dobbie, H., \& Jarvis, W. R. (2002). Vitamin A levels and immunity in humans. Clinical and diagnostic laboratory immunology, 9 (3), 616-621. doi:org/10.1128/cdli.9.3.616-621.2002

Jin, Y. H., Cai, L., Cheng, Z. S., Cheng, H., Deng, T., Fan, Y. P., Fang, C., Huang, D., Huang, L. Q., Huang, Q., Han, Y., Hu, B., Hu, F., Li, B. H., Li, Y. R., Liang, K., Lin, L. K., Luo, L. S., Ma, J., \& Ma, L. L., for the Zhongnan Hospital of Wuhan University Novel Coronavirus Management and Research Team, Evidence-Based Medicine Chapter of China International Exchange and Promotive Association for Medical and Health Care (CPAM) (2020). A rapid advice guideline for the diagnosis and treatment of 2019 novel coronavirus (2019-nCoV) infected pneumonia (standard version). Military Medical Research, 7 (1), 4.

Jordão, K. S. D. L. U., Assumpção, D. D., Barros, M. B. D. A., \& Barros Filho, A. D. A. (2021). Consumo de vitamina E e fontes alimentares na dieta de adolescentes: um estudo transversal de base populacional. Revista Paulista de Pediatria, 39.

Kassir R. (2020). Risk of COVID-19 for patients with obesity. Obesity Reviews, 21 (6), 13034. 10.1111/obr.13034

Kaushik, N., Subramani, C., Anang, S., Muthumohan, R., Shalimar, Nayak, B., Ranjith-Kumar, C. T., \& Surjit, M. (2017). Zinc Salts Block Hepatitis E Virus Replication by Inhibiting the Activity of Viral RNA-Dependent RNA Polymerase. Journal of Virology, 91 (21), 754-17. d10.1128/JVI.00754-17

Khoo, A. L., Chai, L., Koenen, H., Joosten, I., Netea, M., \& van der Ven, A. (2012). Translating the role of vitamin D3 in infectious diseases. Critical Reviews in Microbiology, 38 (2), 122-135. 10.3109/1040841X.2011.622716

Koivisto, O., Hanel, A., \& Carlberg, C. (2020). Key Vitamin D Target Genes with Functions in the Immune System. Nutrients, 12 (4), 1140. doi:org/10.3390/nu12041140

Lang, P. O., Samaras, N., Samaras, D., \& Aspinall, R. (2013). How important is vitamin D in preventing infections? Osteoporosis international, 24 (5), 15371553. 10.1007/s00198-012-2204-6

Lima, W. L., Batista, M. C. C., Silvino, V. O., de Moura, R. C., Mendes, I. L., de Moura, M. S. B. ... da Silva Barbosa, A. K. (2020). Importância nutricional das vitaminas e minerais na infecção da COVID-19. Research, Society and Development, 9 (8), e804986103-e804986103.10.33448/rsd-v9i8.6103

Lippi, G., Lavie, C. J., \& Sanchis-Gomar, F. (2020). Cardiac troponin I in patients with coronavirus disease 2019 (COVID-19): Evidence from a metaanalysis. Progress in Cardiovascular Diseases, 63 (3), 390-391. 10.1016/j.pcad.2020.03.001

Mahan, L. K.; Escott-Stump, S.; Raymond, J.L. (2013). Krause: Alimentos, Nutrição E Dietoterapia. (13a ed.), Elsevier.

Martindale, R et al. (2020). Nutrition Therapy in Critically Ill Patients With Coronavirus Disease 2019. Journal of Parenteral and Enteral Nutrition, 44 (7), 1174-1184. 10.1002/jpen.1930

Poveda, V. D. B., Castilho, A. C. B. A., Nogueira, L. D. S., Ferretti-Rebustini, R. E. L., \& Silva, R. D. C. G. (2018). Aferição do volume residual gástrico: retrato da prática clínica de enfermeiros. Revista da Escola de Enfermagem da USP, 52. org/10.1590/s1980-220x2017038803352

Ryan, P. M. D., \& Caplice, N. M (2020). Is Adipose Tissue A Reservoir For Viral Spread, Imunne Activation And Cytokine Amplification In Covid-19. Obesity Reviews. 1 (1), 1-15. doi:10.1002/oby.22843

Schaible, U. E., \& Kaufmann, S. H. (2007). Malnutrition and infection: complex mechanisms and global impacts. PLoS Medicine, 4 (5), e115. 10.1371/journal.pmed.0040115

Scheen, A. J. (2020). Obésité Et Risque De COVID-19 Sévère. Revue Médicale Suisse. 16 (695), 1115-1119. https://www.revmed.ch/RMS/2020/RMS-N695/Obesite-et-risque-de-COVID1 severe\#: :text=Il\%20appara\%C3\%AEt\%20de\%20plus\%20en,intubation\%20et $\% 20$ une $\% 20$ ventilation $\% 20$ assist\%C3\%A9e.

Shi, S., Qin, M., Shen, B., Cai, Y., Liu, T., Yang, F., Gong, W., Liu, X., Liang, J., Zhao, Q., Huang, H., Yang, B., \& Huang, C. (2020). Association of Cardiac Injury With Mortality in Hospitalized Patients With COVID-19 in Wuhan, China. JAMA cardiology, 5 (7), 802-810. org/10.1001/jamacardio.2020.0950 
Research, Society and Development, v. 10, n. 3, e41210313400, 2021

(CC BY 4.0) | ISSN 2525-3409 | DOI: http://dx.doi.org/10.33448/rsd-v10i3.13400

Shittu, M. O., \& Afolami, O. I. (2020). Improving the efficacy of Chloroquine and Hydroxychloroquine against SARS-CoV-2 may require Zinc additives - A better synergy for future COVID-19 clinical trials. Le Infezioni in Medicina, 28 (2), 192-197.

Skalny, A. V., Rink, L., Ajsuvakova, O. P., Aschner, M., Gritsenko, V. A., Alekseenko, S. I., Svistunov, A. A., Petrakis, D., Spandidos, D. A., Aaseth, J., Tsatsakis, A., \& Tinkov, A. A. (2020). Zinc and respiratory tract infections: Perspectives for COVID-19 (Review). International journal of molecular medicine, 46 (1), 17-26. 10.3892/ijmm.2020.4575

Sociedade Brasileira de Nutrição Parenteral e Enteral (SBNPE). Associação Brasileira de Nutrologia (2011). Terapia Nutricional no paciente com Doença Pulmonar Obstrutiva Crônica. Projeto Diretrizes. São Paulo.

Szklo, S.A. (2020). Associação entre Fumar e Progressão para Complicações Respiratórias Graves em Pacientes com COVID-19. Revista Brasileira de Cancerologia, 66 (2), e-03974. 10.32635/2176-9745.RBC.2020v66n2.974

Wang, D., Hu, B., Hu, C., Zhu, F., Liu, X., Zhang, J., Wang, B., Xiang, H., Cheng, Z., Xiong, Y., Zhao, Y., Li, Y., Wang, X., \& Peng, Z. (2020a). Clinical Characteristics of 138 Hospitalized Patients With 2019 Novel Coronavirus-Infected Pneumonia in Wuhan, China. JAMA, 323 (11), 1061-1069. 10.1001/jama.2020.1585

Wang, Y., Chen, Y., \& Qin, Q. (2020b). Unique epidemiological and clinical features of the emerging 2019 novel coronavirus pneumonia (COVID-19) implicate special control measures. Journal of Medical Virology, 92 (6), 568-576. 10.1002/jmv.25748

Zhang, L. \& Liu, Y. (2020). Potential interventions for novel coronavirus in China: A systematic review. Journal of Medical Virology, 92 (5), 479-490. $10.1002 / \mathrm{jmv} .25707$

Zheng, Y. Y., Ma, Y. T., Zhang, J. Y., \& Xie, X. (2020). COVID-19 and the cardiovascular system. Nature Reviews Cardiology, 17 (5), $259-260$. $10.1038 / \mathrm{s} 41569-020-0360-5$

Zhou, F., Yu, T., Du, R., Fan, G., Liu, Y., Liu, Z., Xiang, J., Wang, Y., Song, B., Gu, X., Guan, L., Wei, Y., Li, H., Wu, X., Xu, J., Tu, S., Zhang, Y., Chen, H., \& Cao, B. (2020). Clinical course and risk factors for mortality of adult inpatients with COVID-19 in Wuhan, China: a retrospective cohort study. Lancet, 395 (10229), 1054-1062. 10.1016/S0140-6736(20)30566-3

Zuo, T., Zhang, F., Lui, G., Yeoh, Y. K., Li, A., Zhan, H., Wan, Y., Chung, A. C. K., Cheung, C. P., Chen, N., Lai, C. K. C., Chen, Z., Tso, E. Y. K., Fung, K. S. C., Chan, V., Ling, L., Joynt, G., Hui, D., Chan, F., Chan, P., \& Ng, S. C. (2020). Alterations in Gut Microbiota of Patients With COVID-19 During Time of Hospitalization. Gastroenterology, 159 (3), 944-955. 10.1053/j.gastro.2020.05.048 